\title{
Parallel processing of two disjunctive targets
}

\author{
MARTIN ARGUIN \\ Université de Montréal, Montréal, Québec, Canada \\ and Laboratoire Théophile Alajouanine, Centre Hospitalier Côte-des-Neiges, \\ Montréal, Québec, Canada \\ and \\ PATRICK CAVANAGH \\ Université de Montréal, Montréal, Québec, Canada
}

\begin{abstract}
We examined the capabilities of preattentive processing by asking subjects to report the relative location of two visual targets, each placed in a separate group of stimuli. In the first experiment, the two targets differed from their distractors on the same feature dimension (shape or color) or on different dimensions, one distinguished by its form and the other by its color. Reaction times (RTs) suggest that the two targets were processed simultaneously (i.e., in parallel). Indeed, the overall processing time was as long when two feature dimensions were involved as the longest processing time for either dimension alone. These results imply that the preattentive processing of several stimuli can occur simultaneously along at least two different feature dimensions. In the second and third experiments, when the target in one group of stimuli was used as the distractors in the other group, RTs increased significantly over RTs in conditions in which the distinctive feature of the targets was unique in the display. In none of these conditions did RTs vary with the number of distractors. These results indicate that although both targets are processed simultaneously when the distinctive features of the targets are unique in the display, two parallel searches (one for each target) must be performed sequentially when there is an overlap between the target in one group of stimuli and distractors in the other. The notion of location as a separate feature dimension is also discussed.
\end{abstract}

Numerous authors have distinguished between the parallel and serial processing of a stimulus array in a visual search task. Support has been found for both types, depending on the stimuli used (Beck \& Ambler, 1973; Bergen \& Julesz, 1983; Egeth, Jonides, \& Wall, 1972). Treisman and her collaborators (Treisman, 1977, 1985; Treisman \& Gelade, 1980; Treisman \& Souther, 1985) have provided evidence that targets differing from distractors by a single feature (e.g., shape or color; disjunctive targets) are detected by the parallel processing of all stimuli, whereas targets defined by a conjunction of features (conjunctive targets) are detected by serial processing. That is, detection time for a disjunctive target remains constant, whatever the number of distractors presented with it, whereas detection time for a conjunctive target increases with the number of distractors. From these results, Treisman and her collaborators concluded that the primitive attributes of visual stimuli are encoded in parallel but that the detection of conjunctions of features requires serial processing by focused attention.

In the experiments cited above, the subjects had to detect the presence of a single target on each trial. What

This research was supported by Natural Sciences and Engineering Research Council of Canada Grant A8606 to P.C. Address correspondence to Martin Arguin, Département de psychologie, Université de Montréal, C.P. 6128, Succ. A, Montréal, Québec H3C 3J7, Canada. happens when subjects are required to detect two or more disjunctive targets in order to perform a given task? Can several targets be processed simultaneously or are they identified one at a time? Two types of experiments have addressed these questions and have yielded contradictory results.

First, an experiment by Duncan (1985) did not support the independence of the identification of multiple targets. He first showed that $d^{\prime}$ for the detection of a tilted line within vertical lines did not vary as a function of the number of distractors. When two targets were present in the display and both had to be reported, however, there was an important decrease in $d^{\prime}$. Whether this reduction in $d^{\prime}$ with multiple targets reflects a shift to sequential target processing or merely less efficient parallel searches (one for each target) is not clear from his results.

On the other hand, Sagi and Julesz (1985) did find evidence for the simultaneous processing of multiple targets. Their subjects were instructed to report the number of vertical and horizontal lines that were presented within a field of oblique lines. The results showed that the proportion of correct responses as a function of stimulus onset asynchrony between the stimulus display and a patterned mask did not vary with the number of target elements.

Sagi and Julesz's (1985) results show that multiple targets can be processed simultaneously, at least when all the targets differ from distractors on the same feature 
dimension (line orientation). The question that interests us is whether simultaneous processing of more than one target occurs when completely different discriminations must be made for their detection-for example, when one target differs from distractors in form and the other in color.

This question is related to the distinction between spatial and functional parallelism. ${ }^{1}$ Spatial parallelism refers to the capacity to detect a target presented in a spatially distributed field of distractors within a time period that is independent of the number of distractors. As mentioned previously, Treisman and her collaborators (Treisman, 1977, 1985; Treisman \& Gelade, 1980; Treisman \& Souther, 1985) have demonstrated that a spatially parallel search occurs for targets distinguished from distractors by a single feature (disjunctive targets). On the other hand, functional parallelism refers to the capacity to encode the different feature dimensions present in a visual display simultaneously. The literature on this topic is contradictory. Some authors have found evidence suggesting serial encoding of feature dimensions (e.g., Egeth, 1966), whereas others have reported evidence favoring parallel encoding (Biederman \& Checkosky, 1970; Downing \& Gossman, 1970; Hawkins, 1969; Saraga \& Shallice, 1973). Finally, Nickerson (1967) claimed that his results could be interpreted in terms of either a serial or a parallel model.

In the experiments reported here we examined whether two spatially separate disjunctive targets could be processed simultaneously and whether this might occur when each of the two targets was distinguished from distractors on a different feature dimension (form and color). Results providing a positive answer to both of these questions would imply more than spatial and functional parallelism as defined here, in that they would indicate simultaneous, spatially separate, parallel searches involving different feature dimensions.

The task used in these experiments required the subjects to report the relative location of two disjunctive targets, each placed in a separate group of stimuli. Thus, a single response required that both targets be located.

\section{EXPERIMENT 1}

The subjects had to report the relative location of two targets, each belonging to a different group of stimuli (Figure 1). Targets differed from their distractors by a single feature, which could be either shape or color. The distractors were always identical in both groups. In two conditions, both targets were identical, differing from distractors either in form (1D-F) or color (1D-C). In another condition, one target differed from distractors by its form and the other by its color (2D). If the two disjunctive targets were processed simultaneously, the reaction times (RTs) in condition 2D should be no longer than the longest RTs for either 1D condition. That is, processing would be complete only when both targets were located, the time needed being determined by the target that took the longest to locate.

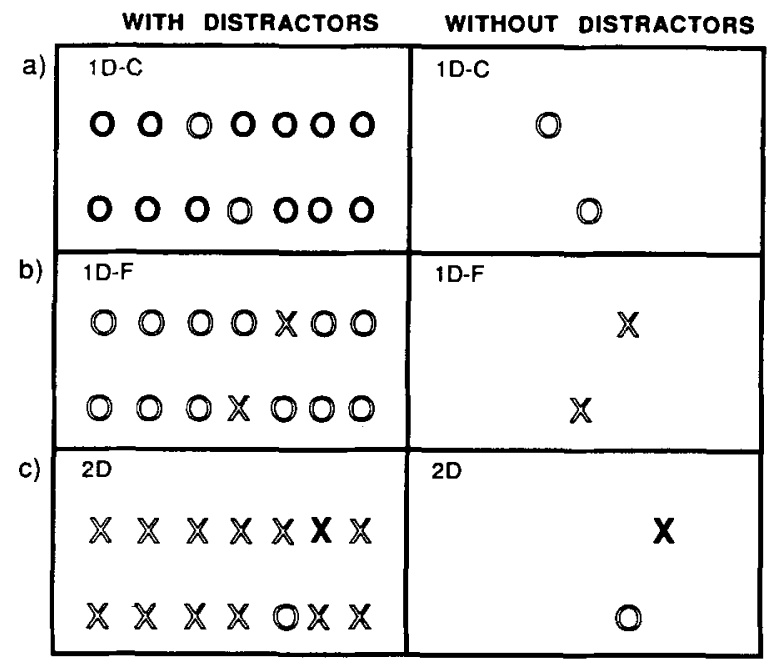

Figure 1. Examples of conditions used in Experiment 1. Targets have the same probabilities in conditions with and without distractors. (a) One dimension with and without distractors. When distractors are present, both targets (outlined $O s$ ) differ from distractors in color (1D-C). (b) One dimension with and without distractors. When distractors are present, both targets (outlined Xs) differ from distractors in form (1D-F). (c) Two dimensions with and without distractors. When distractors are present, one target differs from distractors in form (outlined $O$ ) and the other in color (bold X) (2D). Original colors of stimuli: bold $=$ green, outlined $=$ red.

Conditions in which no distractors were presented with the targets were used as a control to determine whether the presence of a feature difference between targets, as in condition 2D, had an effect on RTs, independently of feature discriminations.

\section{Method}

Subjects. Nine men and 9 women, aged from 18 to 35 , voluntarily took part in the experiment and were paid $\$ 3$ for their collaboration. The only restriction for eligibility was that subjects have normal or corrected-to-normal acuity and normal color vision.

Stimuli. The stimuli were presented on a color video screen placed $166 \mathrm{~cm}$ from the subject; the experiment was controlled by an Apple II microcomputer. The stimuli were the letters $X$ and $O$, each $0.73^{\circ}$ of visual angle high and $0.53^{\circ}$ wide. The two letters were composed of an equal number of pixels and could be either red or green, presented on a black background. Any of the four stimuli available could be a target or a distractor; this was determined randomly. The subjects adjusted the colors to approximate equiluminance by using the minimum flicker technique (mean luminance $=5.26 \mathrm{~cd} / \mathrm{m}^{2}$ ). The fixation point was a filled red square of the same size as the letters.

Two targets were presented above and below the fixation point at a vertical distance of $4.43^{\circ}$ from each other, forming the endpoints of an imaginary line. This line had an orientation of $75^{\circ}$ or $105^{\circ}$ from the horizontal. The eccentricity of this line from the fixation point was set randomly, with the constraint that it be in the central $5^{\circ}$ of visual field. The distractors were placed in two horizontal rows, separated vertically by a distance of $4.43^{\circ}$. In these rows, each $7.80^{\circ}$ from end to end, seven letters (the target and six distractors) were randomly distributed, with the constraint that the space between each one was not less than $0.36^{\circ}$ and not more than $0.64^{\circ}$. There was always one target in each row and all the distractors used 
were identical. Displays in the control conditions without distractors were identical except for the absence of distractors.

Procedure. The subjects were asked to identify the orientation of a virtual line whose two endpoints were defined by the targets. For conditions with distractors, the targets were described to the subjects as the stimuli that differed from the others in their row in form or color.

A trial proceeded as follows. The fixation point was presented in the center of the screen for $500 \mathrm{msec}$, followed by a sound indicating that the subject could press a key in order to start a trial. Following this response, a delay of 1,000 msec preceded the 150msec presentation of the stimuli on the screen. The subject then responded with one of two predetermined keys to indicate the orientation of the virtual line delimited by the targets. The subjects were requested to respond as rapidly as possible while avoiding errors. If a subject did make an error, a buzzer sounded to warn him/her. Trials with RTs below 150 msec or above $2,500 \mathrm{msec}$ were eliminated and replaced later in the session.

Six experimental conditions were used to make a two-factor (distractors $\times$ target type) design. The distractors factor (two levels) corresponded to the presence or absence of distractors in the display. When the targets were presented with distractors, the discrimination the subjects had to perform in order to detect them was varied (three levels). Two of these conditions involved discriminations along a single feature dimension (1D). That is, both targets differed from distractors in either form (1D-F) or color (1D-C). The third condition with distractors involved discriminations along two feature dimensions (2D), one target differing from distractors in form and the other in color. In the 1D conditions, where no distractors were present, the form-versus-color distinction was inoperative, inasmuch as it referred to the feature that distinguished the targets from the (now absent) distractors. In fact, we ran the same condition with twice as many trials as in the other conditions while randomly attributing trials to one of the two ID conditions without distractors. This procedure permitted us to include the no-distractor conditions in a factorial analysis despite the identity of the two 1D conditions without distractors.

All experimental conditions were run in a random order within a single session. The whole test, for each subject, comprised 350 trials, including 30 practice trials. Each session lasted approximately $20 \mathrm{~min}$. The experiment was run in a dark room.

\section{Results}

A $2 \times 3$ analysis of variance (ANOVA) for repeated measures was first applied to the logarithms of the RTs of the correct responses. This analysis showed main effects of distractors $[F(1,17)=52.42, p<.001]$ and target type $[F(2,34)=18.41, p<.001]$, as well as a distractors $\times$ target type interaction $[F(2,34)=14.72$, $p<.001]$. Analysis of simple effects showed the absence of any effect of target type in the absence of distractors $[F(2,34)=2.29$, n.s. $]$, but a significant effect of this tor when distractors were present $[F(2,34)=31.40$, $p<.001]$ (Figure 2). The latter effect stemmed in part from a difference between the 1D-C (color only) and the 2D (form and color) conditions $[t(17)=7.36, p<.001]$. RTs in the 1D-F (form only) and 2D conditions did not differ from each other when distractors were present $[t(17)$ $=1.14$, n.s.].

A similar analysis was performed on error rates, which averaged from $2.72 \%$ to $7.27 \%$, depending on the condition. The pattern of results obtained from this analysis was identical to the one shown by the analysis on RTs. Thus, there were main effects of distractors $[F(1,17)=$

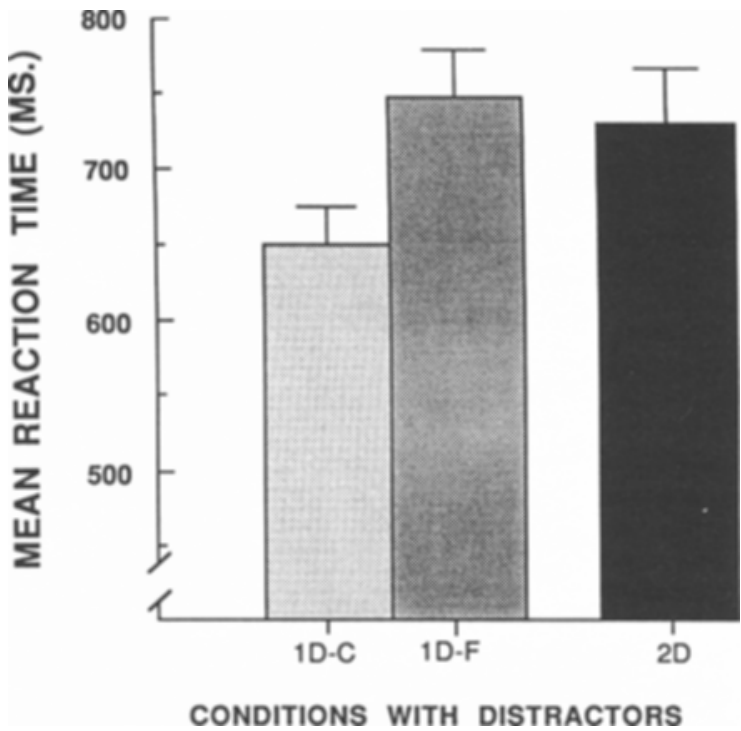

Figure 2. Mean reaction time results for each condition with distractors in Experiment 1. The two columns on the left are for the $1 D$ conditions and the one on the right is for the 2D condition. The thin vertical bars above each column represent the corresponding standard error $(+1 S E)$.

$3.75, p<.05]$ and target type $[F(2,34)=8.33$, $p<.05]$ and an interaction between these two factors $[F(2,34)=3.80, p<.05]$. Analysis of simple effects showed no effect of target type in the absence of distractors $[F(2,34)=1.28, \mathrm{n}$.s. $]$, but a significant effect of this factor when distractors were present $[F(2,34)=7.04$, $p<.01$ ]. In the latter case (Figure 3 ), there was a difference between the error rates in the 1D-C and 2D conditions $[t(17)=3.64, p<.001]$ but not between the 1D$F$ and $2 D$ conditions $[t(17)=1.03$, n.s.]. The correla-

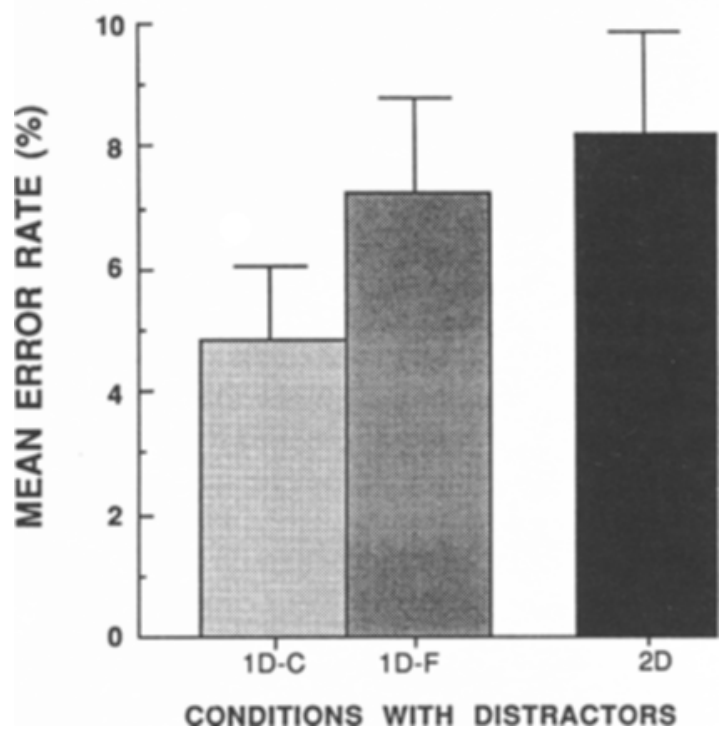

Figure 3. Mean error rates for each condition with distractors in Experiment 1. The conventions are the same as in Figure 2. 
tion between error rates and RTs was positive, indicating that speed-accuracy tradeoff was not a factor in our results.

\section{Discussion}

The results of Experiment 1 indicate that two targets whose distinctive features are unique in the display can be processed simultaneously even when the targets are distinguished from distractors on different feature dimensions. Targets differing from distractors by their form (1D-F) took longer to process than targets distinguished on the basis of their color (1D-C) (see Figure 2). However, RTs for two targets differing in form (1D-F) did not differ from RTs when one target differed from distractors in form and the other in color (2D). These results are thus consistent with the hypothesis of simultaneous parallel searches for the two disjunctive targets, the overall processing time being as long as the longest search time for either target in isolation. This finding also implies that the dimensions of shape and color can be processed in parallel, which agrees with the results of Biederman and Checkosky (1970), Downing and Gossman (1970), Hawkins (1969), and Saraga and Shallice (1973).

An alternative explanation of our results, however, is possible. It might be suggested that instead of searching for both targets simultaneously, the subjects performed two parallel searches, one after the other (i.e., one for each target). Such an explanation would predict that, ideally, RTs in condition 2D should be the average of those in conditions 1D-C and 1D-F, rather than being equal to the longer of the two, as would be the case with parallel processing of both targets. From our data, it seemed difficult to discriminate between the hypothesis of two simultaneous searches and the hypothesis of two sequential parallel searches.

The purpose of Experiments 2 and 3, therefore, was to provide further evidence that the separate groups are processed simultaneously instead of sequentially when subjects perform a task similar to the one used in Experiment 1. In Experiments 2 and 3, subjects still had to report the relative location of two targets; this single response required the processing of both stimuli.

\section{EXPERIMENT 2}

In Experiment 2 we used an experimental paradigm and conditions similar to those of Experiment 1 (Figure 4). However, in one experimental condition, condition $C_{\text {inter }}$ (Figure 4c), each target differed from distractors in its group by a single feature (shape or color) but was identical to the distractors in the other group of stimuli. Therefore, in this condition, targets were defined, relative to the whole display, by a conjunction of location (group) and shape or color.

In related experiments, Treisman (1982) studied visual search in stimulus arrays made up of several groups. The

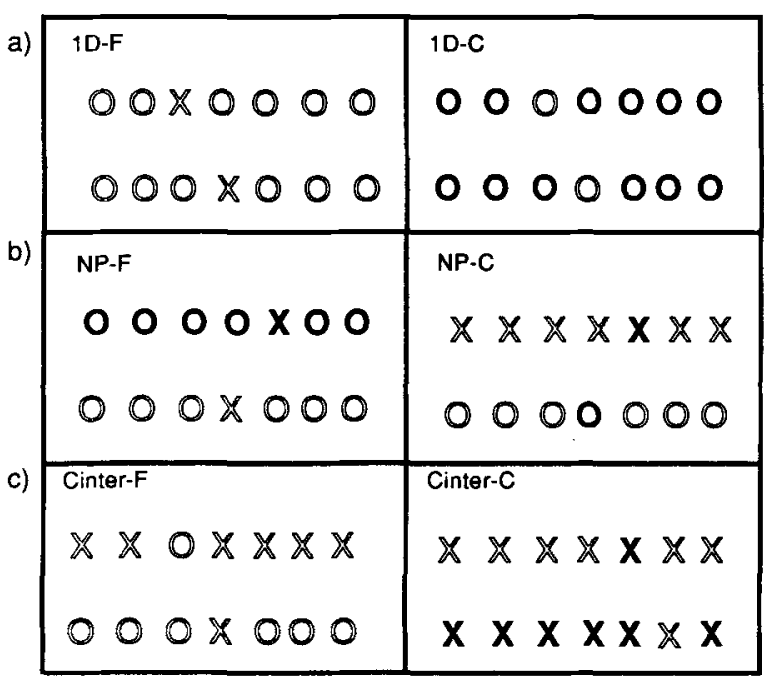

Figure 4. Examples of conditions used in Experiment 2. (a) The groups of stimuli are composed of identical stimuli. 1D-F = Both targets (outlined Xs) differ from distractors in their group in form. 1D-C = Both targets (outlined Os) differ from distractors in their group in color. (b) The groups of stimuli differ in the dimension that is not relevant to target detection. NP-F = Targets (bold and outlined Xs) differ from distractors in their group in shape. NP-C = Targets (bold $X$ and $O$ ) differ from distractors in their group in color. (c) The target in one group of stimuli is used as distractors in the other group. $C_{\text {inter }} F=$ Targets (outlined $O$ and $X$ ) differ from distractors in their group in shape. $C_{\text {inter }}-C=$ Targets (bold and outlined Xs) differ from distractors in their group in color. Original color of stimuli: bold $=$ green, outlined $=$ red.

target to be detected differed from the other stimuli in its group by a single feature, but different from the overall display by a conjunction of shape and color. In these conditions, RTs increased with the number of groups but were independent of the number of stimuli within each group. This implies that subjects searched for the target by sequentially attending to each group but processing each group in parallel. In condition $C_{\text {inter }}$ of the present experiment, as in Treisman's (1982) experiments, each target differed from distractors in its group by a single feature but was defined, relative to the entire display, by a conjunction of features.

It was therefore hypothesized that in condition $\mathrm{C}_{\text {inter }}$ the detection of both targets would require sequential processing of the two groups, and that detection should be slower than in conditions in which the distinctive feature of each target was unique in the display (as in conditions 1D-F, 1D-C, and 2D).

In order to control for the fact that the distractors in the upper and lower rows of stimuli differed from one another in condition $C_{\text {inter, }}$ a new condition, labeled NP, was used (Figure 4b). In this case, one row of stimuli differed from the other on the feature dimension that was not relevant for the target-distractors discrimination. Therefore, the distinctive attribute of the targets in condition NP was still unique in the display. 


\section{Method}

Subjects. Four men and 1 woman, aged from 20 to 39, voluntarily took part in the experiment. The restrictions for eligibility were that the subjects have normal or corrected acuity and normal color vision.

Stimuli. The shapes, colors, display organization, and materials were identical to those of Experiment 1.

Procedure. The task, instructions, and trials were identical to those of Experiment 1.

There were six experimental conditions resulting from the variation of two factors. The first factor was the feature dimension by which targets differed from distractors (dimension; two levels). The dimensions used were form and color. The second factor was the way the two rows of stimuli were constituted relative to each other (grouping; three levels). In two conditions, the distinctive feature of the targets was unique in the display. In these conditions, the groups of stimuli could either be identical to each other (condition 1D) or differ from each other on the dimension that was irrelevant for target detection (e.g., targets differing from distractors in color and groups differing from each other in form; condition NP). In a third condition, the target in one group of stimuli was used as distractors in the other, but targets were still distinguished from distractors in their group by a single feature (condition $\mathrm{C}_{\text {inter }}$ ). Only in this condition were the targets distinguished from the whole display by a conjunction of features.

The six conditions were run in random order within a single session; the type of trial and the distinctive features of the targets were randomly determined. The whole test for each subject comprised 350 trials, including 30 practice trials. Each session lasted approximately $20 \mathrm{~min}$. The experiment was run in a dark room.

\section{Results}

A $2 \times 3$ ANOVA for repeated measures was first applied to the logarithms of the correct RTs (Figure 5). This analysis showed main effects of dimension $[F(1,4)=$ 72.17, $p<.001]$ and grouping $[F(2,8)=11.38$,

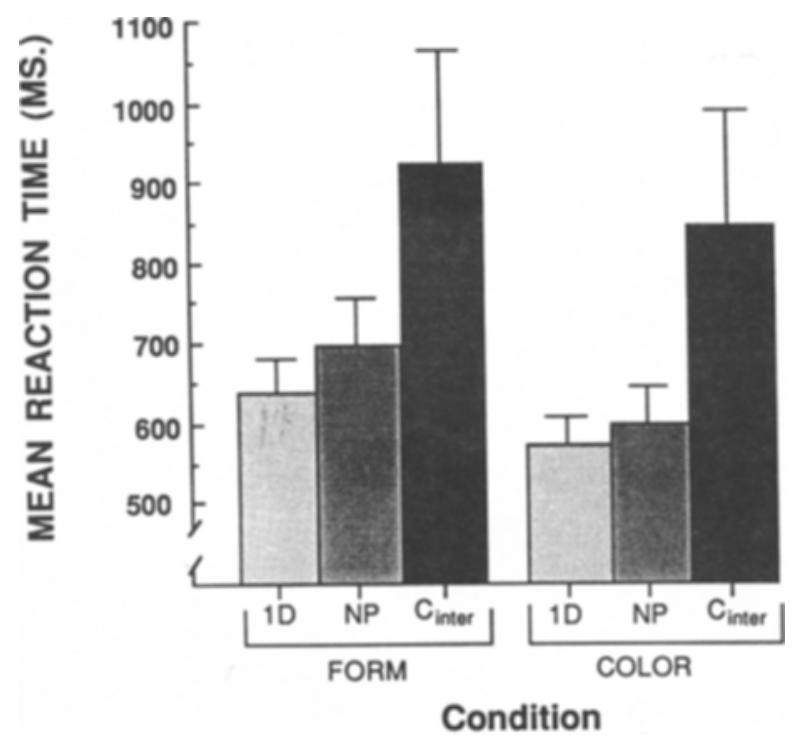

Figure 5. Mean reaction time results for each condition in Experiment 2 . The three columns on the left are for the conditions involving a form discrimination and those on the right are for the conditions involving a color discrimination. The thin vertical bars above each column represent the corresponding standard error (+1SE).

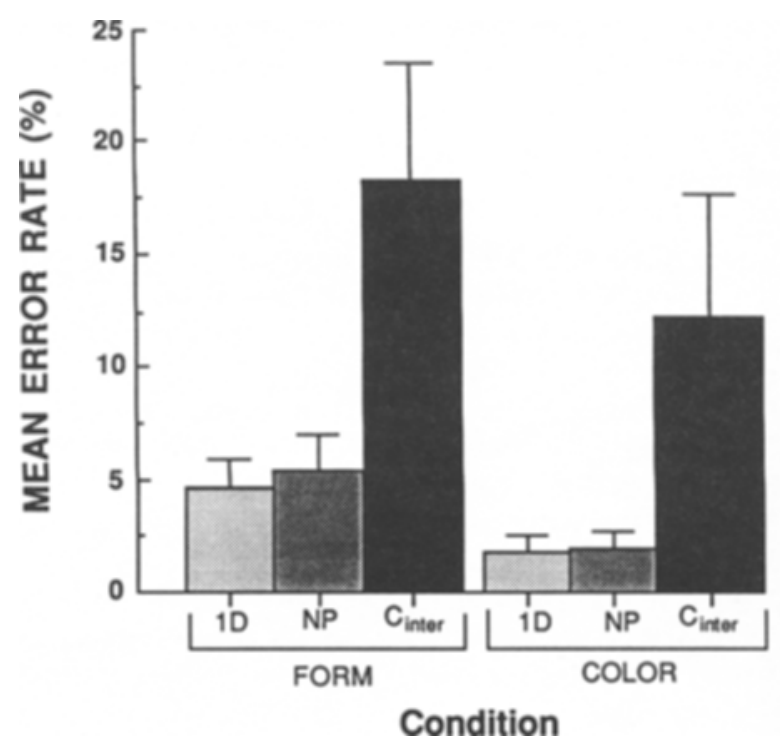

Figure 6. Mean error rates for each condition in Experiment 2. The conventions are the same as in Figure 5.

$p<.005]$. The interaction term was not significant $[F(2,8)<1]$. The main effect of dimension indicates that RTs were longer for targets differing from distractors in form than for those distinguished by color. Mean comparisons on the main effect of grouping, done with the Newman-Keuls test, showed that RTs in conditions 1D and NP did not differ from each other $(p>.05)$, but that they were shorter than those in condition $C_{\text {inter }}$ ( $p<.01$ for 1D and NP).

A similar analysis performed on the error rates showed a pattern of results identical to the one obtained for RTs (Figure 6). Main effects of dimension $[F(1,4)=12.85$, $p<.05]$ and grouping $[F(2,8)=16.89, p<.005]$ were significant, but the dimension $\times$ grouping interaction was not $[F(2,8)<1]$. The main effect of dimension shows that error rates were higher when targets differed from distractors in form than when they differed in color. Mean comparisons indicate that error rates for conditions 1D and NP did not differ from each other $(p>.05)$, but were lower than those in condition $\mathrm{C}_{\text {inter }}(p<.01$ for $1 D$ and NP). The correlation between error rates and RTs was positive.

\section{Discussion}

The main results show that when the target in one group of stimuli was used as distractors in the other group (condition $\mathrm{C}_{\text {inter }}$ ), RTs were markedly higher than in conditions in which the single feature by which the targets differed from distractors was unique in the visual field (conditions 1D and NP).

This increased $\mathrm{RT}$ in condition $\mathrm{C}_{\text {inter }}$ cannot be accounted for by the fact that, in this condition, rows of stimuli differed from one another, since it was also the case in condition NP, where RTs were no longer than in condition 1D. Nor can this result be explained by the fact 
that the specific attributes by which the targets differed from distractors were the same in conditions $1 D$ and NP but not in condition $C_{\text {inter }}$. In condition 2D of Experiment 1 , the two targets were also distinguished from the distractors by different features, but that did not increase RTs.

Therefore, it appears that the reason condition $\mathrm{C}_{\mathrm{inter}}$ required the longest processing time was that in this condition, targets differed from the rest of the display by a conjunction of location (group) and shape or color, whereas in conditions $1 D$ and NP, the feature that made the targets distinct from distractors was unique in the display. This provides support for the hypothesis that two disjunctive targets whose distinctive attributes are unique in the visual field can be processed simultaneously. However, a further test of this hypothesis was necessary, because two different serial processing strategies might account for the longer RTs observed in condition $C_{\text {inter }}$. First, in this condition, the two groups of stimuli might have been processed one at a time, while, within each group, the target was detected by parallel processing. A second possibility would be that in condition $\mathrm{C}_{\text {inter }}$ all stimuli had to be processed in a serial fashion.

\section{EXPERIMENT 3}

The purpose of Experiment 3 was to discriminate between these two possibilities by varying the number of distractors. According to the first explanation (Figure 7a), items within groups are processed in parallel in condition $\mathrm{C}_{\mathrm{inter}}$, and therefore response times in this condition should be independent of the number of distractors. The second explanation (Figure $7 \mathrm{~b}$ ) predicts that RTs in con-

a)

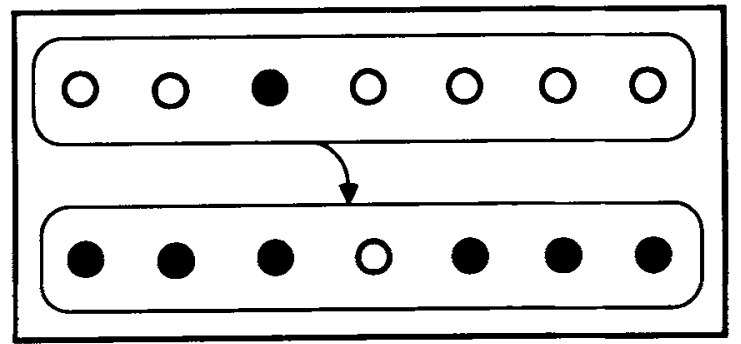

b)

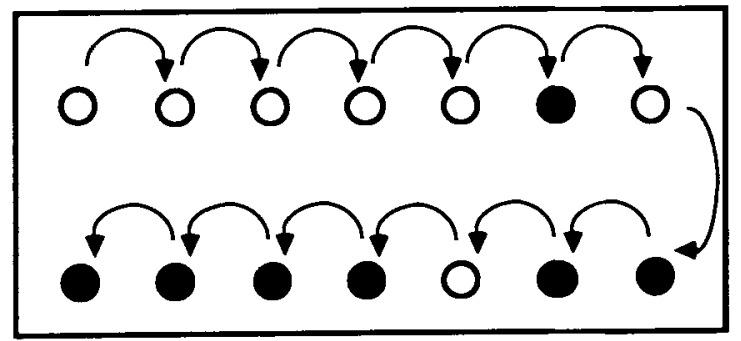

Figure 7. Illustration of the two possible explanations for the results in condition $\mathbf{C}_{\text {imer }}$ of Experiment 2. (a) Processing is parallel within groups but groups are processed sequentially. (b) All stimuli are processed sequentially.
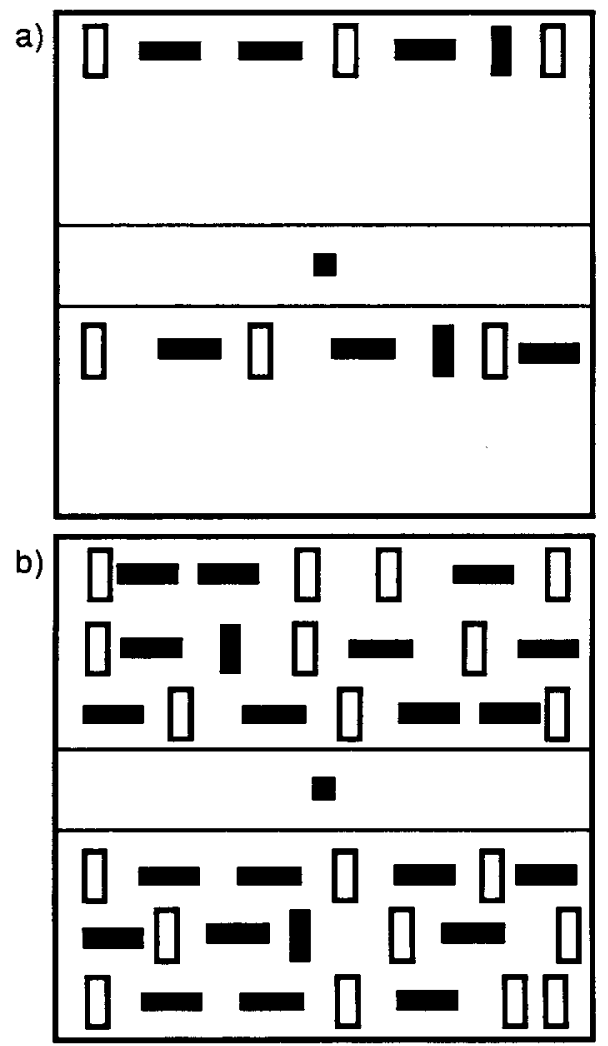

Figure 8. Examples of the stimulus displays used in condition $\mathbf{C}_{\text {intr }}$ of Experiment 3. The disposition of the stimuli was identical in the other conditions. In both (a) and (b), the central dot is the fixation point and the horizontal lines above and below it are used to separate the two groups of stimuli. The targets are bold verticals and differ from distractors in their own group by a conjunction of features (color and orientation). (a) Seven stimuli in each group. (b) Twentyone stimuli in each group. Original color of stimuli: bold = green, outlined $=$ red.

dition $C_{\text {inter }}$ should increase linearly with the number of stimuli in each group.

Only if parallel processing within groups were observed in condition $\mathbf{C}_{\text {intes }}$ could we conclude with certainty that RTs were longer in this condition than in conditions ID and NP (see Figures $4 \mathrm{a}$ and $4 \mathrm{~b}$; the distinctive attribute of the targets is unique in the display) because targets were searched simultaneously in the latter conditions. Otherwise, the sequential group processing strategy depicted in Figure 7a could apply to account for shorter RTs in conditions $1 \mathrm{D}$ and NP than in condition $\mathrm{C}_{\text {inter. }}$

We used a control condition, intended to produce sequential processing within groups, in which targets were distinguished from distractors in their own group by a conjunction of features (Figure 8). In this condition, labeled $\mathrm{C}_{\text {intra, }}$, we expected clear evidence of serial processing within each group.

\section{Method}

Subjects. Four men, aged from 23 to 39 , voluntarily took part in this experiment. All had normal or corrected sight and normal color vision. 
Stimuli. The apparatus used was the same as in Experiments 1 and 2 , and as in those experiments, the subjects were seated at a distance of $166 \mathrm{~cm}$ from the display screen. The stimuli were vertical and horizontal bars made up of an equal number of pixels; the bars could be either red or green and were presented on a black background. The vertical bars were $0.41^{\circ}$ wide and $0.10^{\circ}$ high and the horizontal bars were $0.10^{\circ}$ wide and $0.40^{\circ}$ high. Any of the four stimuli could be a target or a distractor. The subjects adjusted the colors to approximate equiluminance by using the minimum flicker technique (mean luminance $=19.9 \mathrm{~cd} / \mathrm{m}^{2}$ ). The fixation point was a filled gray square (mean luminance $=8.4 \mathrm{~cd} / \mathrm{m}^{2}$ ) $0.35^{\circ}$ wide and $0.24^{\circ}$ high.

Two groups of stimuli were presented on each trial. Each group contained a single target and could consist of one, two, or three rows of seven stimuli each. Within a row, which was $7.40^{\circ}$ from end to end, the seven bars were randomly distributed, with the constraint that the center-to-center distance between bars be not less than $0.95^{\circ}$ and not more than $1.42^{\circ}$.

The location of the targets in each group was selected randomly, with the constraint that both targets be in the same row (upper, middle, or lower) within their respective group and that their horizontal offset be of a single stimulus position. The rows of stimuli in which the targets were placed were presented above and below the fixation point at a vertical distance of $4.07^{\circ}$ from each other, the targets forming the limits of a virtual line. The eccentricity of this line was set randomly, with the constraint that it be in the central $5^{\circ}$ of visual field.

At $0.80^{\circ}$ above and below the fixation point were horizontal gray lines (mean luminance $=8.4 \mathrm{~cd} / \mathrm{m}^{2}$ ) that were $7.93^{\circ}$ long. They were used to isolate the two groups of stimuli from each other.

Procedure. The subjects were asked to identify the orientation of an imaginary line whose two endpoints were defined by the targets. This line had an orientation of $74^{\circ}$ or $106^{\circ}$ from the horizontal. The targets were described to the subjects as the stimuli that were different from the others in their group in color, orientation, or, in condition $\mathrm{C}_{\text {intra }}$, by a conjunction of color and orientation. The whole experiment was run in two sessions, which took place on separate days. All conditions were presented in each session.

Each trial proceeded as in Experiments 1 and 2, with the following exceptions: the fixation point was presented on the screen for $1,000 \mathrm{msec}$ before a sound indicated to the subject that he could press a key in order to start a trial; the delay between this keypress and the presentation of the stimuli was $1,500 \mathrm{msec}$; and the stimuli remained available until a response was made. Trials with RTs below $150 \mathrm{msec}$ were eliminated and replaced later in the session.

The conditions used in Experiment 3 were identical to those of Experiment 2 (see Figure 4), except for three aspects. First, a new condition $\left(C_{\text {intra }}\right)$, in which conjunctive targets were used, was added to the others. Second, a new factor was used: the number of stimuli presented in each group was varied randomly $(7,14$, or 21 ; i.e., one, two, or three rows, respectively). Finally, the dimensions used were orientation and color, rather than shape and color.

In each session, each of the seven conditions ( $\mathrm{C}_{\text {intra }}$ and two levels of dimension, orientation, and color, for each of $1 D, N P, C_{\text {inter }}$ ) was run in a separate block of 30 trials, including 3 practice trials at the beginning of the block and 9 trials for each stimulus-group size. To show the subjects what stimuli to look for (pilot experiments had shown this to be essential for conjunctive targets), the targets were displayed at the left and right of the center of the screen before the beginning of each block. The same targets were used throughout the block, each being randomly assigned to the group above or below the fixation point. The order of blocks was set randomly for each subject in each session.

Each session included a total of 210 trials and lasted approximately $20 \mathrm{~min}$. The experiment was run in a dark room.

\section{Results}

All conditions could not be included in a single factorial design because, in condition $\mathrm{C}_{\text {intra, }}$, the distinction between targets differentiated from distractors in orientation and color was not possible. Therefore, a 2 (dimension) $\times 3$ (grouping; conditions 1D, NP, and $\left.\mathrm{C}_{\text {inter }}\right) \times 3$ (number of stimuli) ANOVA for repeated measures was performed on the logarithms of the correct RTs by using all factors and removing condition $\mathrm{C}_{\text {intra. }}$.

This first analysis showed a main effect of grouping $[F(2,6)=22.62, p<.01]$ and a grouping $\times$ number of stimuli interaction $[F(4,12)=3.62, p<.05]$. No other main effects or interactions reached significance.

Because this first analysis on RTs showed no main effect and no interaction involving the factor of dimension, this factor was eliminated and data for discriminations of orientation and color were pooled. This allowed us to include the $C_{\text {intra }}$ condition in a 4 (grouping) $\times 3$ (number of stimuli) ANOVA for repeated measures in order to compare all the relevant conditions (Figure 9).

This analysis showed a main effect of grouping $[F(3,9)$ $=47.07, p<.001]$, a main effect of number of stimuli $[F(2,6)=39.35, p<.001]$, and an interaction $[F(6,18)$ $=14.91, p<.001]$ between these two factors. The analysis of simple effects indicates that, at all levels of the number of stimuli factor, the effect of grouping was significant $(p<.001)$. Mean comparisons were done with the Newman-Keuls test. With 7 stimuli in each group, RTs in conditions 1D and NP did not differ $(p>.05)$, but they were shorter than those in conditions $C_{i n t e r}$ and $\mathrm{C}_{\text {intra }}\left(p<.01\right.$ for $1 \mathrm{D}$ and NP). RTs in conditions $\mathrm{C}_{\text {inter }}$

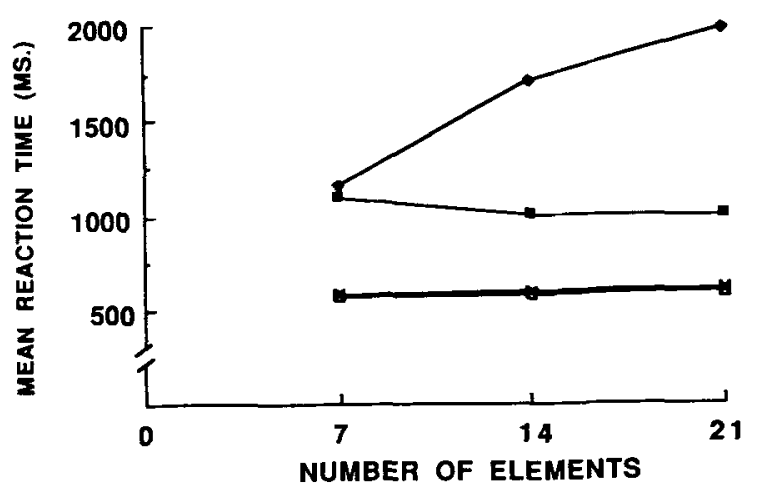

\begin{tabular}{|llll|}
\hline LEGEND & \multicolumn{3}{c|}{ REGRESSION EQUATIONS } \\
\hline$-1 D$ & - & $y=558.02+1.94 x$ & $R=.97$ \\
$\rightarrow$ NP & - & $y=566.33+2.42 x$ & $R=.99$ \\
$-G_{\text {inter }}-$ & $y=1131.69-6.61 x$ & $R=.86$ \\
$\rightarrow C_{\text {intra }}-$ & $y=790.79+59.22 x$ & $R=.98$ \\
\hline
\end{tabular}

Figure 9. Mean reaction time as a function of the number of stimuli. Regression equations and correlation coefifients are given for each condition in Experiment 3. The results for discriminations of orientation and color have been combined. 
and $C_{\text {intra }}$ did not differ $(p>.05)$. With 14 and 21 stimuli in each group, $R T s$ in condition $C_{\text {intra }}$ were longer than those in conditions $\mathrm{C}_{\mathrm{inter}}, 1 \mathrm{D}$, and NP $(p<.01$ for each comparison). As with groups of 7 stimuli, RTs in conditions 1D and NP did not differ $(p>.05)$, and they were shorter than those in condition $\mathrm{C}_{\text {intra }}(p<.01)$.

A regression analysis carried out on the correct RTs for each condition as a function of the number of stimuli showed no increase in RT with the number of distractors for conditions 1D and NP (slopes of 1.94 $[F(2,6)=2.31$, n.s. $]$ and $2.42 \mathrm{msec}$ per item $[F(2,6)=$ 2.95 , n.s.], respectively), a nonsignificant decrease in condition $C_{\text {inter }}$ (slope of -6.61 msec per item $[F(2,6)$ $=3.19$, n.s.]), and a significant increase of $59.22 \mathrm{msec}$ per item $[F(2,6)=23.14, p<.01]$ in condition $C_{\text {intra }}$ (see Figure 9).

A preliminary 2 (dimension) $\times 3$ (grouping) $\times 3$ (number of stimuli) analysis, excluding condition $\mathrm{C}_{\text {intra }}$, was performed on the error rates, as had been done for the RTs. This analysis showed no significant main effects or interactions. When the results of the dimension factor were grouped and the $\mathbf{C}_{\text {intra }}$ condition was added to the grouping factor, a 4 (grouping) $\times 3$ (number of stimuli) ANOVA again showed the absence of any significant effect.

\section{Discussion}

The results of Experiment 3 replicated those of Experiment 2 in that RTs in conditions $1 D$ and NP did not differ from each other but were shorter than those observed in condition $\mathrm{C}_{\text {inter }}$. Also, as predicted by the hypothesis of parallel processing within groups in condition $\mathrm{C}_{\text {inter }}$, there was no increase in RTs as a function of the number of distractors in this condition. Only in condition $\mathrm{C}_{\text {intra }}$ did RTs increase with the number of stimuli presented.

The results for condition $\mathbf{C}_{\text {intra }}$ suggest that, in our experiment, stimuli could be processed sequentially when conditions required it. Therefore, the absence of an effect of the number of stimuli presented with the targets in conditions $1 \mathrm{D}, \mathrm{NP}$, and $\mathrm{C}_{\text {inter }}$ must be interpreted as evidence of parallel search through the items of each group.

An account for the fact that RTs were longer in condition $\mathrm{C}_{\text {inter }}$ than in conditions $1 \mathrm{D}$ and NP must be provided. In light of Treisman's (1982) experiments, the most appropriate interpretation of this result is that there was serial processing of groups but parallel processing of stimuli within groups in condition $C_{\text {inter }}$, whereas both stimulus groups were processed in parallel in conditions ID and NP. We therefore conclude that in conditions $1 D$ and NP, where the distinctive attribute of the targets was unique in the display, two spatially separate parallel searches (one for each target) occurred simultaneously.

\section{GENERAL DISCUSSION}

Overall, the results obtained from the experiments described here suggest the following conclusions about the encoding of visual stimuli.
First, two disjunctive targets can be processed simultaneously if their distinctive attributes are unique in the visual field. Furthermore, these two parallel searches proceed simultaneously even when the two distinctive attributes involve different dimensions, that is, when one target differs from distractors in shape and the other in color. These results are therefore consistent with those of Sagi and Julesz (1985) and extend the realm of preattentive processing to include the simultaneous search of two disjunctive targets when different discriminations must be performed for each target.

Second, it appears that a limiting factor for target detection is the overlap between the target in one group of stimuli and the distractors in the other. In this case, the target may be processed in parallel within its group, but the separate groups of stimuli must be processed sequentially. This type of processing is identical to the one observed by Treisman (1982) for targets distinguished from distractors in their group by a single feature and from the entire display by a conjunction of shape and color. By analogy, targets in our experiments were distinguished by a single feature within their group and by a conjunction of location and another feature relative to the whole display.

Thus our data indicate that conjunctions involving location provided results similar to conjunctions involving other feature dimensions. Therefore, in an extension of Treisman and her collaborators' theory (Treisman \& Gelade, 1980; Treisman \& Souther, 1985), it may be proposed that location can serve as a feature in visual conjunctions, just as the other constituent attributes of visual stimuli (form, color, etc.) can.

This aspect of our results also agrees with Treisman and Souther's (1985) suggestion that stimulus features are coded by representations that are not selective to stimulus location, and that position information is represented separately on a specialized location map that is common to all feature-analyzing pathways. That is, in condition $\mathrm{C}_{\text {inter, }}$, without conjoining location information and information about other stimulus features, a subject could not discriminate which of two identical stimuli was a target and which was a distractor, since the only distinction between them was the group in which each was placed. According to Treisman (Treisman \& Gelade, 1980; Treisman \& Souther, 1985), the perception of conjunctions of features primarily encoded through separate pathways requires attention. Since serial processing of groups was observed in condition $\mathrm{C}_{\text {inter, }}$ which indicates the involvement of attention, it may be proposed that location is encoded by a pathway separate from those coding other stimulus attributes. In fact, if it were otherwise, the results for condition $C_{\text {inter }}$ of Experiments 2 and 3 should not have differed from those for conditions ID and NP, conjunctions involving location and other stimulus attributes being encoded along a single representation without the aid of attention. This was obviously not the case.

If location is in fact encoded by a separate pathway, the results of Experiment 1 suggest, in agreement with Treisman and Souther's (1985) hypothesis, that a single 
location representation (or map) encodes the position of targets independently of the feature dimension along which they differ from distractors. Indeed, if separate location maps existed for targets defined by shape and those defined by color, the results of Experiment 1 should have shown an advantage in RT for conditions involving a single feature dimension, since some transfer time to a common location representation would have been necessary in condition $2 \mathrm{D}$ to allow subjects to make the relative position judgment.

Although our results suggest a separate pathway for the coding of location, evidence from Nissen (1985) and Nissen, Case, and Isenberg (1986) indicates that location may have a special status as a feature dimension. Their results imply that correct information about the location of a given stimulus is necessary to enable the subject to report both its color and its shape; that is, cross-referencing of information from separate pathways coding the various features of a stimulus proceeds through a map in which the location of this stimulus is represented. This view is consistent with Treisman and Souther's (1985) model, which assumes that spatial attention allows the perception of conjunctions of features, these being linked via the location map.

\section{REFERENCES}

BECK, J., A AMBLER, B. (1973). The effects of concentrated and distributed attention on peripheral acuity. Perception \& Psychophysics, 14, 225-230.

Bergen, J. R., \&ulesz, B. (1983). Parallel versus serial processing in rapid pattern discrimination. Nature, 303, 696.

Biederman, I., Checkosxy, S. F. (1970). Processing redundant information. Journal of Experimental Psychology, 83, 486-490.

Downing, B. D., \& Gossman, J. R. (1970). Parallel processing of multidimensional stimuli. Perception \& Psychophysics, 8, 57-60.

Duncan, J. (1985). Visual search and visual attention. In M. I. Pos- ner \& O. S. M. Marin (Eds.), Attention and performance XI (pp. 85105). Hillsdale, NJ: Erlbaum.

EGETH, H. (1966). Parallel versus serial processes in multidimensional stimulus discrimination. Perception \& Psychophysics, 1, 245-252.

EgeTh, H., Jonides, J., \& Wal, S. (1972). Parallel processing of multielement displays. Cognitive Psychology, 3, 674-698.

Hawxins, H. L. (1969). Parallel processing in complex visual discrimination. Perception \& Psychophysics, 5, 56-64.

Nickerson, R. S. (1967). "Same" -"different" response times with multiattribute stimulus differences. Perceptual \& Motor Skills, 24, 543-554.

Nissen, M. J. (1985). Accessing features and objects: Is location special? In M. I. Posner \& O.S.M. Marin (Eds.), Attention and performance $X I$ (pp. 205-219). Hillsdale, NJ: Erlbaum.

NisSen, M. J., CASE, L., \& Isenberg, L. (1986). Attentional processing and the independence of colors and shape. Bulletin of the Psychonomic Society, 24, 349. (Abstract)

SAGI, D., Julesz, B. (1985). "Where" and "what" in vision. Science, 228, 1217-1219.

Saraga, E., Shallice, T. (1973). Parallel processing of the attributes of single stimuli. Perception \& Psychophysics, 13, 261-270.

Treisman, A. (1977). Focused attention in the perception and retrieval of multidimensional stimuli. Perception \& Psychophysics, 22, 1-11. Treisman, A. (1982). Perceptual grouping and attention in visual search for features and for objects. Journal of Experimental Psychology: Human Perception \& Performance, 8, 194-214.

Treisman, A. (1985). Preattentive processing in vision. Computer Vision \& Image Processing, 31, 156-177.

Treisman, A., Gelade, G. (1980). A feature-integration theory of attention. Cognitive Psychology, 12, 97-136.

Treisman, A., SouTHER, J. (1985). Search asymmetry: A diagnostic for preattentive processing of separable features. Journal of Experimental Psychology: General, 114, 285-310.

\section{NOTE}

1. We thank an anonymous reviewer for pointing out this distinction between spatial and functional parallelism.

(Manuscript received April 7, 1987; revision accepted for publication November 19, 1987.) 